\title{
Baricitinib for the treatment of rheumatoid arthritis
}

\author{
Ivan Urits ${ }^{1}$, Jacob Israel ${ }^{2}$, Hayk Hakobyan², George Yusin², Grace Lassiter², Nathan Fackler², \\ Amnon A. Berger ${ }^{1}$, Hisham Kassem ${ }^{3}$, Alan Kaye ${ }^{4}$, Omar Viswanath ${ }^{5,6,7}$
}

\begin{abstract}
${ }^{1}$ Department of Anesthesiology, Critical Care, and Pain Medicine, Beth Israel Deaconess Medical Center, Harvard Medical School, Boston, Massachusetts, United States

${ }^{2}$ Georgetown University School of Medicine, Washington, District of Columbia, United States

${ }^{3}$ Department of Anesthesiology, Mount Sinai Medical Center, Miami Beach, Florida, United States

${ }^{4}$ Department of Anesthesiology, Louisiana State University Health Shreveport, Shreveport, Los Angeles, United States

${ }^{5}$ Valley Anesthesiology and Pain Consultants - Envision Physician Services, Phoenix, Arizona, United States

${ }^{6}$ Department of Anesthesiology, University of Arizona College of Medicine Phoenix, Phoenix, Arizona, United States

${ }^{7}$ Department of Anesthesiology, Creighton University School of Medicine, Omaha, Nebraska, United States
\end{abstract}

\begin{abstract}
Rheumatoid arthritis (RA) is a common inflammatory disease with several implications on health, disability and economy. Conventional treatment for RA centers on anti-inflammatory drugs and specific targeting of tumor necrosis factor $\alpha$ (TNF- $\alpha$ ) and interleukin 6 (IL-6). Baricitinib is a novel, Food and Drug Administration (FDA) approved, once daily oral drug that is effective in combination with current treatment and results in significantly reduced symptoms with good safety profile. Further studies are required to find rare side effects and evaluate the long term efficacy in disease modulation and patient symptom reduction. This is a comprehensive review of the literature on baricitinib for the treatment of RA.

This review provides an update on the pathophysiology, diagnosis and conventional treatment of RA, then proceeds to introduce baricitinib and the data that exists to support or refute its use in RA. The presented study also indicated clinical trials confirming the effectiveness of baricitinib in this indication.
\end{abstract}

Key words: rheumatoid arthritis, novel treatment, baricitinib, Janus kinase/signaling transducer and activator of transcription, kinase inhibitor.

\section{Introduction}

Rheumatoid arthritis (RA) is the most common autoimmune inflammatory arthritis, affecting roughly $0.5-1.0 \%$ of the population [1-6]. Rheumatoid arthritis is a chronic disease that can attack the joints of the hands, wrists, elbows, shoulders, knees, and ankles [1]. This joint damage is progressive and irreversible [1]. This autoimmune disease may cause general symptoms such as: fatigue, fever, and weakness; the inflammation can also affect other organs, particular the cardiovascular system increasing risk for myocardial infarction $[1,7,8]$. Rheumatoid arthritis leads to reduce functional capacity, productivity, and quality of patient's life [5, 9]. It occurs roughly 2.5 times as frequently in women as men, hinting that hormones, environmental factors, and genetics may play a role in its development and can affect any age group $[1,6,10,11-16]$.

While many aspects of RA pathophysiology are still unknown, modern scientific advances suggest that the primary cause of RA is deregulation of JAK/STAT pathway. Janus Kinases (JAK) is a type of tyrosine kinase that alters transcription and translation processes by delivering signals from the extracellular environment to the nucleus. These kinases promote gene expression by phosphorylating appropriate sequences in the nucleus,

Address for correspondence:

Hisham Kassem, Department of Anesthesiology, Mount Sinai Medical Center, 4300 Alton Rd, Miami Beach, 33140 Florida, United States, e-mail: hkassem.md@gmail.com

Submitted: 7.05.2020; Accepted: 4.12.2020 
which further causes transcriptional activation triggered by signal transducers and activators of transcription (STAT) [17].

Abnormal activity of interleukin 6 (IL-6) is thought to be one of the primary causes of RA, since it plays an important role in directing T-cells to the tissues, thereby causing inflammation. Inhibition of IL- 6 and of the tumor necrosis factor (TNF- $\alpha$ ) is shown to be one of the key mechanisms dictating the design of modern RA drugs. Tumor necrosis factor significantly affects bone metabolism and renewal of bony tissues by altering osteoclast activity [18]. Further research has postulated other genetic mechanisms of RA development [19-27].

\section{Baricitinib}

Recently the two innovative companies introduced baricitinib on the pharmaceutical market. Company's website briefly describes the mechanism of action, stating that it functions as a JAKi and inhibits responses to cellular messenger proteins called cytokines, which are thought to be the key triggers of inflammation and swelling associated with RA [28].

According to the manufacturers websites, baricitinib is a tablet recommended once a day by adults to treat moderate to severe active RA. Due to a number of potential serious side effects, it is typically prescribed as a second choice drug after patients have tried taking TNF inhibitors, but did not feel significant relief or could not tolerate the medicine due to side effects [28]. Both brands, have warnings about potential serious side effects. Both of them also warn against using the medication in the case of a known serious infection, such as tuberculosis (TB), and therefore advise performing TB screening before starting the course of treatment. Other side effects may affect renal, cardiovascular, and eye health $[20,28]$.

\section{Food and Drug Administration approval}

Baricitinib became Food and Drug Administration (FDA) approved in 2018 [29]. It has been approved in Europe and Japan in $2 \mathrm{mg}$ and $4 \mathrm{mg}$ doses [21]. It only can be indicated if previous therapies with methotrexate or other TNF inhibitors have failed. Serious side effects related to immunosuppression are concerning. Thus, Food and Drug Administration approved $2 \mathrm{mg}$ dose and did not approve the $4 \mathrm{mg}$ dose, with the latter possibly being more effective but causing more pronounced side effects [22]. Researchers also warn that baricitinib may lead to cancer, thrombosis, or hyperlipidemia in certain patient groups $[23,30]$.

\section{Mechanism of action}

Baricitinib acts upon Janus kinases by inhibiting and preventing them from activating factors leading to gene expression [17, 31]. These kinases generally trigger STAT pathway, thus starting the cascade of transcription initiation of effector genes [31]. This process, in turn, triggers the autoimmune and inflammatory reactions associated with main symptoms of RA [17, 31]. A complete and accurate mechanism for the last step is still being studied and clarified.

It was shown that baricitinib primarily targets and inhibits JAK1 and JAK2 and has less efficacy in inhibiting TYK2 and JAK3 [31]. Typically, patients with RA will have elevated circulating B-lymphocytes, T-lymphocytes, macrophages, as well as elevated levels of the common arrays of immunoglobulins. In particular, baricitinib inhibits JAK1 and JAK2.

It was also shown to inhibit IFN- $\gamma$ and IL- 6 through a series of pathways. This drug is actively filtrated in the glomeruli of kidneys and consequently secreted. Interestingly, CYP3A4, which is an enzyme involved in sequestering and eliminating toxins and drugs from the body, metabolizes a very small fraction (less than 10\%) of baricitinib in the body [17].

Recent study suggests that the granulocyte-macrophage colony-stimulating factor (GM-CSF) mediated cellular signals are inhibited by JAKi, which, in turn, provides significant relief of RA symptoms.

The article also gives strong evidence that IL- 6 is one of the major contributors to RA-associated inflammatory response, followed by joint damage and other RArelated complications. The study further states that IL-10, IFN- $\alpha$ and IFN- $\gamma$ play a crucial role in RA pathophysiology. However, inhibition of IL-10 may not be recommended due to the fact that overall action of IL-10 may in fact reduce inflammation [31].

Mitchell et al. [32] provide evidence that JAKi hamper the rate of chemotaxis and diapedesis of neutrophils towards IL-8, one of the important inflammatory markers in RA, thus alleviating the symptoms. However, these drugs have not been shown to increase the rate of apoptosis in neutrophil colonies taken from patients with RA, which causes them to remain active for a longer period of time. In addition, part of the inflammatory cascade generated by neutrophils also involves production of reactive oxygen species (ROS), which could not be suppressed by JAKi either.

Thus, many RA drugs, including baricitinib, are unable to fully quench the inflammatory response. It is thought that neutrophils taken from RA patients are already primed in vivo to cause inflammation and cannot be adjusted by medications [32]. Therefore, future studies can further elucidate the underlying mechanisms 
and possible solutions to reverse the increased production of ROS in RA cases.

Baricitinib intake was associated with lower amounts of neutrophils in the blood, therefore leading to decreased inflammation. Several Interleukin types were shown to promote proliferation of lymphocytes, which can also be controlled by the inhibition of JAK3. Baricitinib treatment also produced elevated levels of low-density lipoprotein cholesterol (LDL), high-density lipoprotein cholesterol (HDL), and creatinine [33, 34]. Since JAK2 plays a role in controlling hematopoiesis, its inhibition by baricitinib resulted in decreased hemoglobin levels [33].

However, some papers note that no significant changes in leukocyte count or any other drastic abnormalities were noted following the course of administration of baricitinib $[35,36]$. This discrepancy is possibly due to different characteristics of patient populations and needs to be explored further.

\section{Clinical trials}

Since the FDA approval of baricitinib in May 2018, a number of randomized controlled trials have demonstrated the efficacy and safety profile of baricitinib in treating rheumatoid arthritis.

In 2014 two randomized controlled trials were conducted in healthy volunteers to demonstrate the pharmacokinetics and safety of baricitinib [37]. Multiple ascending doses between $1 \mathrm{mg}$ and $20 \mathrm{mg}$ were studied. The results showed dose-linear and time-invariant pharmacokinetics with insignificant effects from a highfat diet. The plasma concentration of baricitinib peaks 1.5 hours after oral ingestion and mean renal clearance is $11.8 \mathrm{l} / \mathrm{h}$. This study also demonstrated a dose related decline in absolute neutrophil count.

\section{Phase II clinical trials}

In 2015 a phase IIb trial investigated the efficacy of baricitinib at $1 \mathrm{mg}, 2 \mathrm{mg}, 4 \mathrm{mg}$, or $8 \mathrm{mg}$ vs. placebo [38]. The study involved 301 patients from 69 institutions in 9 countries who had failed prior treatment with methotrexate. The primary endpoint was the proportion of study volunteers in the $4 \mathrm{mg}$ or $8 \mathrm{mg}$ cohort that received a positive result on the American College of Rheumatology 20\% (ACR20) score at 12 weeks. The ACR2O is a tool that uses multiple measures to objectively evaluate improvement in rheumatoid arthritis symptoms. This trial showed a significant response to treatment in the combined $4 \mathrm{mg}$ or $8 \mathrm{mg}$ baricitinib cohort as compared to placebo ( $76 \%$ vs. $41 \%$ respectively) at 12 weeks of therapy.

Additionally, baricitinib was well tolerated by most of the study participants, and all baricitinib groups showed an increased response compared to placebo in the other secondary endpoints, including ACR50, ACR70, and remission.

At 12 weeks the baricitinib and placebo groups experienced similar proportions of treatment-emergent adverse events. The mean neutrophil count declined in all baricitinib cohorts, but there was no significant decline in mean lymphocyte count as compared to the placebo. Mean low-density lipoproteins and mean high-density lipoproteins also increased in all the baricitinib cohorts in comparison to placebo. This study shows the effectiveness of baricitinib treatment in patients with active rheumatoid arthritis who were unresponsive to methotrexate therapy.

The inhibition of IL- 6 signaling by baricitinib may in part explain how the drug affects lipoprotein metabolism and particle distribution, and was explored in a separate analysis of this trial in 2017 [39]. Changes in lipoprotein particle size and particle number, as well as changes in lipid profile, were assessed at weeks 12 and 24 in association with clinical efficacy.

Interestingly, the study found that following 2 weeks of baricitinib, patients experienced dose-related increases in serum lipid levels. Increases in HDL cholesterol, LDL cholesterol, and triglyceride levels were observed and remained elevated through 24 weeks. The observed increase in LDL cholesterol was associated with a shift in LDL particle size to large LDL particles [39].

Furthermore, treatment with baricitinib resulted in an increase in apolipoprotein $\mathrm{A}-\mathrm{I}$ and a reduction in the serum amyloid A (SAA) content of HDL particles, which rendered HDL particles more efficient for reverse cholesterol transport.

These increased HDL cholesterol levels correlated with improved clinical outcomes at 12 weeks, as evidenced by improvement in Disease Activity Score 28-joint assessment using the Simplified Disease Activity Index [SDAI] and C-reactive protein level (DAS28-CRP) [39]. This association provides support for a potential relationship between increases in HDL levels and reduction of disease activity scores and inflammation in patients with rheumatoid arthritis. No such relationship was observed in patients treated with placebo.

Further evaluation of this trial using population pharmacokinetic/pharmacodynamics models to determine dose/exposure-response relationships was performed to assess the efficacy and safety of different doses of baricitinib for a potential phase III trial in the future [40].

The primary efficacy endpoint assessed was the ACR20/50/70 and the primary safety endpoint assessed was anemia. The results of the study showed a faster onset of ACR2O improvement in the $4 \mathrm{mg}$ and $8 \mathrm{mg}$ dosed groups along with a slight increase in incidence of anemia for the $8 \mathrm{mg}$ dose group. 
The evaluation concluded stating that $4 \mathrm{mg}$ QD was the optimal dosing from a risk-benefit standpoint, and that $2 \mathrm{mg}$ QD may potentially be efficacious, however this should be explored further in a phase III trial. Importantly, this study also found no benefit to BID dosing from a safety and efficacy standpoint.

Another phase IIb randomized control trial in 2016 demonstrated similar effectiveness of baricitinib treatment in Japanese patients with rheumatoid arthritis who were concurrently taking methotrexate [41]. The study enrolled 145 patients. The primary endpoint was the ACR20 response rate of patients taking $4 \mathrm{mg}$ or $8 \mathrm{mg}$ daily of baricitinib compared to placebo at 12 weeks. A significantly greater proportion of patients in the $4 \mathrm{mg}$ or $8 \mathrm{mg}$ group responded to treatment relative to placebo ( $77 \%$ vs. $31 \%$ ) at 12 weeks.

Improvement of symptoms including remission and physical function was demonstrated as early as 2 weeks in the $4 \mathrm{mg}$ and $8 \mathrm{mg}$ group. The adverse effects of baricitinib treatment were limited. The $1 \mathrm{mg}, 2 \mathrm{mg}$, and $4 \mathrm{mg}$ baricitinib group showed similar rates of adverse events as compared to placebo.

However, there was a slightly increased rate of adverse events and abnormal laboratory values in the $8 \mathrm{mg}$ group. There were 3 serious adverse events (SAEs) reported during the study period: cholecystitis in the placebo group, acute pancreatitis in the $2 \mathrm{mg}$ baricitinib group, and cataract in the $8 \mathrm{mg}$ baricitinib group. It is unclear if these SAEs were related to the experimental drug.

The safety of baricitinib in this Japanese cohort showed similar results to other trials with non-Japanese patients, with no malignancies, serious infection, tuberculosis, pneumocystis pneumonia, herpes zoster cases, or Gl perforations reported. This trial justifies the need to further study the benefit to risk ratio of $2 \mathrm{mg}$ and $4 \mathrm{mg}$ baricitinib treatment in patients with rheumatoid arthritis concurrently taking methotrexate.

This 12-week study was then extended to a 64-week study [42]. In this extension, patients that were originally randomized to placebo, $1 \mathrm{mg}$, or $2 \mathrm{mg}$ doses were re-randomized to either $4 \mathrm{mg}$ or $8 \mathrm{mg}$ doses following the conclusion of the 12-week study. However, after analysis of data from other phase II trials occurring at this time, a decision was made to switch patients from $8 \mathrm{mg}$ to $4 \mathrm{mg}$, so that all patients were taking $4 \mathrm{mg}$ of baricitinib by the conclusion of the extension study. Of the $142 \mathrm{pa}-$ tients who completed the 12-week study, 109 of these patients continued and completed the extension study. During the extension period, patients who were originally assigned to the placebo group noted a significant improvement in ACR20, while those originally treated with baricitinib successfully maintained or improved on the progress made in the first 12 weeks.
No changes in safety of baricitinib were noted with a longer period of treatment. Notably, although no incidences of herpes zoster were noted in the first 12 weeks of the study, 11 patients (7.8\%) developed herpes zoster during the extension and dropped out of the study. This was the most common reason for discontinuation of the study overall.

While this may seem remarkable, reactivation of herpes zoster is a common side effect of disease-modifying antirheumatic drug (DMARD) therapy and it is even recommended by the American College of Rheumatology for patients to get the herpes zoster vaccine prior to starting DMARD therapy. This trial highlights the beneficial effects of baricitinib in patients concurrently taking methotrexate and shows no difference in safety and efficacy between short- and long-term dosing of baricitinib.

\section{A phase III RA-BEACON trial}

A phase III RA-BEACON trial in 2016 involving 527 patients compared the use of $2 \mathrm{mg}$ or $4 \mathrm{mg}$ of daily baricitinib with placebo at 24 weeks [43]. The primary endpoint used the ACR20 response to determine clinical improvement at 12 weeks. The study population consisted of patients $>18$ years old with moderate-to-severe RA who had discontinued prior treatment with conventional tumor necrosis factor inhibitors (TNFis) or biologic disease-modifying antirheumatic drugs (bDMARDs) due to insufficient response or intolerance after $>3$ months [44]. The results showed significantly more patients in the $4 \mathrm{mg}$ baricitinib group had an ACR20 response than the placebo group (55\% vs. 27\%). Additionally, the $4 \mathrm{mg}$ group as compared to the placebo showed a difference in response to the HAQ-DI and DAS28-CRP score, but not the SDAI score.

The rates of adverse events at 24 weeks were higher among the $2 \mathrm{mg}$ and $4 \mathrm{mg}$ group (71\% and 77\%) relative to the placebo (64\%). The most common cause of adverse events were infections, including respiratory infections, bronchitis, and urinary tract infections. However, the rates of serious adverse events were similar throughout 24 weeks: 4\% in the placebo group, 10\% in the $2 \mathrm{mg}$ group, and $7 \%$ in the $4 \mathrm{mg}$ group. The two baricitinib treatment groups were also associated with a drop in neutrophil count versus placebo at 24 weeks $(-560,-630$ vs. +130$)$. The serum creatinine and LDL levels were already elevated in the baricitinib groups compared to placebo. These results show evidence of clinical improvement with baricitinib treatment in a study population with disease particularly refractory to multiple biologic therapies.

Furthermore, the efficacy of baricitinib demonstrated in RA-BEACON was reflected by clinically significant 
changes in patient reported outcomes (PROs). These are core measures established by the American College of Rheumatology that assess disease activity in clinical trials and may improve the patient-physician relationship by addressing patient concerns such as onset of drug action, sustainability or risk of relapse, impact on quality of life, and efficacy plateau.

Patient reported outcomes utilize standardized scales (PROs) measuring fatigue, duration of morning joint stiffness, severity of worst joint pain, physical and mental quality of life, Short Form-36, work productivity and impairment of daily activities, and physical function. RA-BEACON used a minimum clinically important difference (MCID) to assess the clinical relevance of changes to the aforementioned scores. Of note, baseline PROs were similar across treatment groups and represented significant disease burden. PROs were assessed at baseline as well as weeks 1, 2, 4 and every 4 weeks thereafter to week 24 [44]. Over 24 weeks, the majority of PROs significantly improved among patients receiving baricitinib compared with placebo, with patients receiving baricitinib $4 \mathrm{mg}$ demonstrating a faster and greater magnitude of change than the baricitinib $2 \mathrm{mg}$ group. In addition, the PRO improvements were not influenced by the type or number of previous bDMARDs used.

Furthermore, the groups receiving baricitinib reported significant improvement in morning joint stiffness (MJS) duration and fatigue as well as in regular activity (WPAI-RA), EQ-5D scores, and pain when compared to placebo. In contrast, no significant differences were observed for the SF-36 MCS measure between patients treated with baricitinib compared with placebo [44].

A post-hoc analysis was performed following the RA-BEACON trial to examine sub-groups of the patient population and determine if history of prior bDMARD use had an effect on the efficacy and safety of baricitinib [45]. The study examined ACR20s in multiple sub groups of the 527 patients and found no interactions with age, weight, geographic region, disease duration, seropositivity, corticosteroid use, number of prior bDMARDs used, number of prior TNF inhibitors used, or type of TNF inhibitors used, with $2 \mathrm{mg}$ or $4 \mathrm{mg}$ baricitinib. Notably, the only change in safety was an increased risk of infections and serious adverse events in patients who had taken three or more bDMARDs prior to baricitinib treatment. However, the small number of patients in the subgroup with a history of three or more bDMARD use limits the significance of this finding.

\section{A phase III RA-BUILD trial}

The phase III RA-BUILD studied the effectiveness of $2 \mathrm{mg}$ or $4 \mathrm{mg}$ of baricitinib therapy compared to placebo in 684 randomized patients who also did not respond to conventional synthetic disease-modifying antirheumatic drugs (csDMARD) [46]. The study population was naïve biologic DMARD treatment. The primary endpoint was the ACR20 at 12 weeks. Most of the study population was receiving simultaneous CSDMARD therapy and only $7 \%$ of the patients were not taking concomitant cSDMARD therapy. A statistically significant increase in the ACR20 response rate for patients taking $4 \mathrm{mg}$ of baricitinib (62\%) compared to placebo (39\%) was seen at 12 weeks. There was no difference in treatment effect of patients taking concomitant csDMARDs or no csDMARD. Additionally, a reduction in radiographic progression of structural joint damage was observed at 24 weeks in both groups receiving baricitinib therapy relative to placebo. There was no significant difference in the rate of adverse events between both baricitinib groups (67\%, $71 \%)$ and placebo (71\%).

The baricitinib groups showed an increased incidence of low neutrophil count and elevated LDL and HDL values. However, similar rates of serious infections were observed in placebo, $2 \mathrm{mg}$ and $4 \mathrm{mg}$ baricitinib groups $(2 \%,<1 \%$, and $2 \%)$. Both baricitinib groups also exhibited a small increase in the serum creatinine. The results of this trial demonstrated symptomatic relief and a beneficial effect on joint damage without significant side effects in patients who failed to benefit from prior csDMARD therapy.

\section{A phase III RA-BEAM trial}

In 2017 the RA-BEAM phase III trial was the first trial to investigate the use of baricitinib versus another biologic, adalimumab, in patients worldwide taking background methotrexate therapy [47]. Adalimumab is an anti-tumor necrosis factor a monoclonal antibody, a biologic that was used as standard of care in combination with methotrexate for patients with moderate to severe RA. The primary endpoint was the response to ACR20 at 12 weeks. A significant increase in the response rate was demonstrated in $4 \mathrm{mg}$ of daily baricitinib use compared to placebo (70\% vs. 40\%) as well as compared to $40 \mathrm{mg}$ of adalimumab (70\% vs. 61\%). Structural joint damage was also decreased at 24 weeks in both the baricitinib and adalimumab treatment groups relative to placebo. Adverse events through week 24 occurred at a rate of $60 \%$ in the placebo group, $71 \%$ in the baricitinib group, and $68 \%$ in the adalimumab group. The baricitinib and adalimumab groups both exhibited a decrease in neutrophil count and an elevation in aminotransferase, creatinine, LDL, and HDL levels as compared to placebo.

This groundbreaking trial showed the superiority of baricitinib plus methotrexate to what was considered standard of care treatment at the time, adalimumab plus methotrexate. 


\section{A phase III RA-BEGIN trial}

The 52-week phase III RA-BEGIN trial was the first to investigate the difference between methotrexate monotherapy, baricitinib monotherapy, and the combination of baricitinib and methotrexate therapy [48]. The primary endpoint assessed the ACR20 response rate at 24 weeks between baricitinib monotherapy and methotrexate monotherapy. At 24 weeks the response rate of baricitinib monotherapy (77\%) was higher than methotrexate monotherapy (62\%). Symptomatic improvement was observed in the baricitinib group as quickly as 1 week in comparison to methotrexate alone.

Similar results to baricitinib monotherapy was seen in baricitinib plus methotrexate (MTX) combination therapy. The baricitinib plus MTX group showed significant superior radiographic benefit relative to MTX monotherapy, but not baricitinib monotherapy. At 52 weeks the rates of serious adverse events were similar between MTX monotherapy, baricitinib monotherapy, and baricitinib plus MTX combination therapy $(10 \%, 8 \%$, and $8 \%$, respectively). There were more non-serious infections recorded in the baricitinib plus MTX group than either baricitinib or MTX monotherapy.

This trial supported the known benefits of MTX monotherapy in RA patients, while demonstrating superior outcomes in patients taking baricitinib alone or in combination with MTX. Additional benefit in radiographic evidence and measures of inflammation was shown in baricitinib plus MTX compared to baricitinib alone. However, combination therapy increased the risk of adverse events, such as non-serious infection.

Furthermore, RA-BEGIN utilized PROs to assess the safety and efficacy of baricitinib in adults with moderateto-severe RA. The study found comparable effects on PRO improvements in the baricitinib monotherapy and baricitinib +MTX groups when compared to MTX monotherapy, with both baricitinib regimens appearing consistently more effective than MTX alone. Over 24 weeks, the majority of PROs significantly improved among patients receiving baricitinib compared with placebo, with patients receiving baricitinib $4 \mathrm{mg}$ demonstrating a faster and greater magnitude of change than the baricitinib $2 \mathrm{mg}$ group. In addition, the PRO improvements were not influenced by the type or number of previous bDMARDs used. Interestingly, the majority of PRO measures of joint pain, tiredness, duration of MJS, pain, fatigue, physical function, HRQOL, and PtGA improved to a greater extent in many or all time points measured in patients in both baricitinib groups compared to MTX monotherapy [48].

Furthermore, the groups receiving baricitinib reported significant improvement in MJS duration and fatigue as well as in regular activity (WPAI-RA), EQ-5D scores, and pain when compared to placebo. In contrast, no significant differences were observed for the SF-36 MCS measure between patients treated with baricitinib compared with placebo [44]. These statistically significant improvements in PROs suggest that baricitinib alone or combined with MTX may be an alternative therapy for patients for whom MTX monotherapy is undesirable.

Of note, a post-hoc analysis was performed following phase 3 of the RA-BEGIN study evaluating structural damage progression (as measured via radiographs of the hands and feet at weeks 12, 24 and 52) between each of the treatment groups [49]. The study looked particularly at two markers for RA disease activity, the Disease Activity Score for 28-joint count with serum high-sensitivity C-reactive protein (DAS28-hSCRP) and Simplified Disease Activity Index (SDAI). Typically, acceptable management of RA symptoms is indicated by a DAS28CRP score $<3.2$ and a SDAl score $<11$. This study found that if patients were able to stay below those thresholds, the risk of structural damage was lower in those treated with baricitinib alone or combination therapy with MTX. In patients with a DAS28-CRP > 3.2 or a SADI > 11, only combination therapy with MTX and baricitinib was sufficient to decrease the risk of structural damage.

The results of the four phase three trials assessing the efficacy and safety of baricitinib (RA-BEGIN, RA-BEAM, RA-BUILD, and RA-BEACON) showed significant improvement in ACR20 with $4 \mathrm{mg}$ baricitinib when compared to placebo, MTX, and adalimumab standard of care treatment at 12 or 24 weeks. A subgroup analysis was performed in July of 2018 to see if the 394 Japanese patients treated across these trials showed similar benefit to treatment as the general study population [50]. The authors of this subgroup analysis found that across all four trials, equivalent improvements were made in ACR2O in Japanese patients when compared to the overall study population. The study did highlight differences in the Japanese population from other participants in these studies, including a lower body weight and lower average dose of MTX. However, an equal response to baricitinib was noted across all trials despite these differences.

The authors concluded that while this data is promising, long-term effects of baricitinib in this study population have yet to be published, noting that 328 of the 394 Japanese patients are enrolled in the 84 month long RA-BEYOND study that is expected to conclude in 2024.

\section{Trials testing drug-drug interactions}

Baricitinib undergoes active renal tubular secretion, andispredominantlyeliminatedfromthebodyunchanged in urine [51]. Baricitinib's secretion is dependent on the basolaterally expressed OAT3 transporter and the apically expressed P-GP, BRCP, and MATE2-K transporters [51]. 
In the presence of probenecid, a strong OAT3 inhibitor, $\mathrm{CL}_{\mathrm{r}}$ and $\mathrm{CL} / \mathrm{F}$ of baricitinib decreased $69 \%$ and $51 \%$ respectively, and the $\mathrm{AUC}_{(0-\infty)}$ of baricitinib doubled in healthy subjects [51].

Additionally, diclofenac and ibuprofen, OAT3 inhibitors with less inhibition potential than probenecid, were evaluated using physiologically based pharmacokinetic (PBPK) modeling and in vitro inhibition data to predict the inhibition potential for the OAT3 mediated secretion of baricitinib. The aforementioned study used the in vitro IC50 value of $4.4 \mu \mathrm{M}$ to reproduce the renal clearance of baricitinib and the inhibitory effect of probenecid. Using ibuprofen and diclofenac in vitro IC50 values of $4.4 \mu \mathrm{M}$ and $3.8 \mu \mathrm{M}$ toward OAT3, 1.2 and $1.0 \mathrm{AUC}_{(0-\infty)}$ ratios of baricitinib were predicted, suggesting that $\mathrm{Co}$-administration of diclofenac or ibuprofen is safe as it does not cause clinically relevant drug-drug interactions with baricitinib [51]. These predictions are relevant for furthering our understanding of the safety profile of new RA therapies.

To sum up baricitinib, currently a second line drug for RA, is a novel oral drug that is based on the above mentioned research. It is a once-a-day drug that is orally available and targets the JAK/STAT signaling pathway by acting as a JAK inhibitor. It is approved in Europe, Japan and the US for treatment of RA after failure of treatment with methotrexate (MTX) and TNF inhibitors. Acting upstream in the JAK/STAT pathway, baricitinib inhibits specifically JAK1 and JAK2 and decreases the expression of IL- 6 and TNF- $\alpha$, as well as other pro-inflammatory cytokines such as IL-10, IL-8, IFN- $\alpha$. While decreasing inflammation, baricitinib interferes with other pathways involved with JAK/STAT signaling and may induce elevated levels of LDL and HDL and decreased hemoglobin production.

Phase I and II trials conducted so far proved the safety and efficacy of baricitinib in RA, including with concurrent use with MTX. The accepted dose currently is that of daily $4 \mathrm{mg}$ which appears to balance the side effects (anemia, hypercholesterolemia, re-activation of latent infection) with the benefit (as measured by ACR20) and improvement in patients' symptoms at 12-weeks. The RA-BEACON Phase III trial re-iterated these findings, found no safety increased in $2 \mathrm{mg}$ over $4 \mathrm{mg}$ dosing and proved that $4 \mathrm{mg}$ dosing significantly improved symptoms after 12-weeks of treatment, as measured by ACR20. Post-hoc analysis pointed to extensive prior treatment as an increased risk of latent infection reactivation, but otherwise could not point to any other safety or efficacy factors.

The RA-BUILD tested $2 \mathrm{mg}$ and $4 \mathrm{mg}$ dosing and used ACR20 at 12-weeks for end point. The results, while not as impressive as RA-BEACON, similarly demonstrat- ed significant relief of symptoms in the baricitinib over placebo, with or without concurrent DMARD use. Importantly, this study also showed decreased joint damage at the 24-weeks mark. RA-BEAM and RA-BEGIN compared baricitinib to MTX and other biologics and found some evidence to support its used over alternative treatments and not just in addition to current DMARD treatment.

\section{Conclusions}

There is convincing evidence to support the use of baricitinib in RA, especially in addition to current treatment with DMARD. It does carry significant risks, and those will have to be weighed against its efficacy. Phase IV and aftermarket data is still required to determine any long term risks of using baricitinib, the long term effectiveness of this treatment, and rare potential side effects. It is also still unclear how baricitinib use affects joint damage in the long term and whether or not its main effect is on disease symptoms or not. Further research into the pathophysiology of RA will likely present even further opportunity to find treatment targets in this common inflammatory disease.

The authors declare no conflict of interest.

\section{References}

1. Lee DM, Weinblatt ME. Rheumatoid arthritis. Lancet 2001; 358: 903-911, DOI: 10.1016/S0140-6736(01)06075-5.

2. Singh JA, Saag KG, Bridges SL Jr, et al. 2015 American College of Rheumatology guideline for the treatment of rheumatoid arthritis. Arthritis Rheumatol 2016; 68: 1-26, DOI: 10.1002/art.39480.

3. Yelin E, Wanke LA. An assessment of the annual and long-term direct costs of rheumatoid arthritis: the impact of poor function and functional decline. Arthritis Rheum 1999; 42: 12091218, DOI: 10.1002/1529-0131(199906)42:6<1209::AID-ANR18> 3.0.CO;2-M.

4. Helmick CG, Felson DT, Lawrence RC, et al. Estimates of the prevalence of arthritis and other rheumatic conditions in the United States. Part I. Arthritis Rheum 2008; 58: 15-25, DOI: 10.1002/art.23177.

5. Pincus T, Callahan LF, Sale WG, et al. Severe functional declines, work disability, and increased mortality in seventy-five rheumatoid arthritis patients studied over nine years. Arthritis Rheum 1984; 27: 864-872, DOI: 10.1002/art.1780270805.

6. Scott DL, Wolfe F, Huizinga TW. Rheumatoid Arthritis. Lancet 2010; 376: 1094-1108, DOI: 10.1016/S0140-6736(10)60826-4.

7. Myasoedova E, Davis JM 3rd, Crowson CS, Gabriel SE. Epidemiology of rheumatoid arthritis: Rheumatoid arthritis and mortality. Curr Rheumatol Rep 2010; 12: 379-385, DOI: 10.1007/s11926010-0117-y.

8. Solomon DH, Karlson EW, Rimm EB, et al. Cardiovascular morbidity and mortality in women diagnosed with rheumatoid arthritis. Circulation 2003; 107: 1303-1307, DOI: 10.1161/01. cir.0000054612.26458.b2. 
9. Sullivan PW, Ghushchyan V, Huang XY, Globe DR. Influence of rheumatoid arthritis on employment, function, and productivity in a nationally representative sample in the United States. J Rheumatol 2010; 37: 544-549, DOI: 10.3899/jrheum.081306.

10. van der Woude D, van der Helm-van Mil AHM. Update on the epidemiology, risk factors, and disease outcomes of rheumatoid arthritis. Best Pract Res Clin Rheumatol 2018; 32: 174187, DOI: 10.1016/j.berh.2018.10.005.

11. Hemminki K, Li X, Sundquist J, Sundquist K. Familial associations of rheumatoid arthritis with autoimmune diseases and related conditions. Arthritis Rheum 2009; 60: 661-668, DOI: 10.1002/art.24328.

12. Silman AJ, Newman J, MacGregor AJ. Cigarette smoking increases the risk of rheumatoid arthritis: Results from a nationwide study of disease-discordant twins. Arthritis Rheum 1996; 39: 732-735, DOI: 10.1002/art.1780390504.

13. Liao KP, Alfredsson L, Karlson EW. Environmental influences on risk for rheumatoid arthritis. Curr Opin Rheumatol 2009; 21: 279-283, DOI: 10.1097/BOR.0b013e32832a2e16.

14. Spector TD. Epidemiology of the rheumatic diseases. Curr Opin Rheumatol 1993; 5: 132-137, DOI: 10.1097/00002281199305020-00002

15. Lee DM, Schur PH. Clinical utility of the anti-CCP assay in patients with rheumatic diseases. Ann Rheum Dis 2003; 62: 870-874, DOI: 10.1136/ard.62.9.870.

16. Sauerland U, Becker H, Seidel M, et al. Clinical utility of the anti-CCP assay: experiences with 700 patients. Ann N Y Acad Sci 2005; 1050: 314-318, DOI: 10.1196/annals.1313.033.

17. Bechman K, Yates M, Galloway JB. Corrigendum to "The new entries in the therapeutic armamentarium: The small molecule JAK inhibitors" [Pharmacol. Res. 147 (2019) 104392]. Pharmacol Res 2020; 153: 104634, DOI: 10.1016/j.phrs.2020.104634.

18. McInnes IB, Schett G. Pathogenetic insights from the treatment of rheumatoid arthritis. The Lancet 2017; 389: 23282337, DOI: 10.1016/S0140-6736(17)31472-1.

19. Wayne JS. Load partitioning influences the mechanical response of articular cartilage. Ann Biomed Eng 1995; 23: 40-47, DOI: 10.1007/BF02368299.

20. Baricinix (baricitinib) - Beacon Medicare Limited http://www. baricinix.com/.

21. Kunwar S, Collins CE, Constantinescu F. Baricitinib, a Janus kinase inhibitor, in the treatment of rheumatoid arthritis: a systematic literature review and meta-analysis of randomized controlled trials. Clin Rheumatol 2018; 37: 2611-2620, DOI: 10.1007/s10067-018-4199-7.

22. Salaffi F, Carotti M, Di Carlo M, et al. High-resolution computed tomography of the lung in patients with rheumatoid arthritis. Medicine (Baltimore) 2019; 98: e17088, DOI: 10.1097/ MD.0000000000017088.

23. Mogul A, Corsi K, McAuliffe L. Baricitinib: the second FDA-approved JAK inhibitor for the treatment of rheumatoid arthritis. Ann Pharmacother 2019; 53: 947-953, DOI: 10.1177/1060028019839650

24. Gupta A, Pipe SG, Towheed T, Anastassiades T. Is rheumatoid arthritis a risk factor for fractures: a systematic review of observational studies. Curr Rheumatol Rev 2020; 16: 29-37, DOI: 10.2174/1573397115666190723160312.
25. Gauri LA, Singh U, Kapur S, et al. Study of APOE Gene and D2S439 marker in patients of rheumatoid arthritis and their correlation with severity of disease: a case control study. J Assoc Physicians India 2019; 67: 43-48.

26. Naidu G, Bhilave N, Sharma K, et al. Prevalence of metabolic syndrome in rheumatoid arthritis patients: a case control study from a Tertiary Care Centre in North India. J Assoc Physicians India 2019; 67: 22-24.

27. Cheng T, Wang $M$, Chen $L$, et al. Increased expression of CD40/TRAF1 and activation of nuclear factor-kB-dependent proinflammatory gene expression in collagen-induced arthritis. Scand J Rheumatol 2018; 47: 455-460, DOI: 10.1080/ 03009742.2018.1432684.

28. What is Olumiant? Olumiant (baricitinib) https://www.olumiant.com/what-is-olumiant.

29. Mayence A, Vanden Eynde JJ. Baricitinib: a 2018 Novel FDAapproved small molecule inhibiting Janus kinases. Pharmaceuticals (Basel) 2019; 12: 1-7, DOI: 10.3390/ph12010037.

30. Qiu C, Zhao X, She L, et al. Baricitinib induces LDL-C and HDL-C increases in rheumatoid arthritis: a meta-analysis of randomized controlled trials. Lipids Health Dis 2019; 18: 1-11, DOI: 10.1186/s12944-019-0994-7.

31. McInnes IB, Byers NL, Higgs RE, et al. Comparison of baricitinib, upadacitinib, and tofacitinib mediated regulation of cytokine signaling in human leukocyte subpopulations. Arthritis Res Ther 2019; 21: 183, DOI: 10.1186/s13075-019-1964-1.

32. Mitchell TS, Moots RJ, Wright HL. Janus kinase inhibitors prevent migration of rheumatoid arthritis neutrophils towards interleukin-8, but do not inhibit priming of the respiratory burst or reactive oxygen species production. Clin Exp Immunol 2017; 189: 250-258, DOI: 10.1111/cei.12970.

33. Wu Z-P, Pei Z, Bai J-Z, et al. Efficacy and safety of baricitinib for active rheumatoid arthritis in patients with an inadequate response to conventional synthetic or biological disease modifying anti rheumatic drugs: A meta analysis of randomized controlled trials. Exp Ther Med 2018; 16: 2449-2459, DOI: 10.3892/etm.2018.6495.

34. Taylor PC, Keystone EC, van der Heijde D, et al. Baricitinib versus placebo or adalimumab in rheumatoid arthritis. N Engl I Med 2017; 376: 652-662, DOI: 10.1056/NEJMoa1608345.

35. Tanaka Y, McInnes IB, Taylor PC, et al. Characterization and changes of lymphocyte subsets in baricitinib-treated patients with rheumatoid arthritis an integrated analysis. Arthritis Rheumatol 2018; 70: 1923-1932, DOI: 10.1002/art.40680.

36. Smolen JS, Genovese MC, Takeuchi T, et al. Safety profile of baricitinib in patients with active rheumatoid arthritis with over 2 years median time in treatment. J Rheumatol 2019; 46: 7-18, DOI: 10.3899/jrheum.171361.

37. Shi JG, Chen X, Lee F, et al. The pharmacokinetics, pharmacodynamics, and safety of baricitinib, an oral JAK $1 / 2$ inhibitor, in healthy volunteers. J Clin Pharmacol 2014; 54: 1354-1361, DOI: $10.1002 / j c p h .354$.

38. Keystone EC, Taylor PC, Drescher E, et al. Safety and efficacy of baricitinib at 24 weeks in patients with rheumatoid arthritis who have had an inadequate response to methotrexate. Ann Rheum Dis 2015; 74: 333-340, DOI: 10.1136/annrheumdis-2014-206478. 
39. Kremer JM, Genovese MC, Keystone E, et al. Effects of baricitinib on lipid, apolipoprotein, and lipoprotein particle profiles in a Phase Ilb study of patients with active rheumatoid arthritis. Arthritis Rheumatol 2017; 69: 943-952, DOI: 10.1002/art.40036.

40. Zhang X, Chua L, Ernest C 2nd, et al. Dose/exposure-response modeling to support dosing recommendation for Phase III development of baricitinib in patients with rheumatoid arthritis. CPT Pharmacometrics Syst Pharmacol 2017; 6: 804-813, DOI: 10.1002/psp4.12251.

41. Tanaka Y, Emoto K, Cai Z, et al. Efficacy and safety of baricitinib in Japanese patients with active rheumatoid arthritis receiving background methotrexate therapy: a 12-week, double-blind, randomized placebo-controlled study. J Rheumatol 2016; 43: 504-511, DOI: 10.3899/jrheum.150613.

42. Tanaka Y, Ishii T, Cai Z, et al. Efficacy and safety of baricitinib in Japanese patients with active rheumatoid arthritis: a 52-week, randomized, single-blind, extension study. Mod Rheumatol 2018; 28: 20-29, DOI: 10.1080/14397595.2017.1307899.

43. Genovese MC, Kremer J, Zamani O, et al. Baricitinib in patients with refractory rheumatoid arthritis. N Engl J Med 2016; 374 1243-1252, DOI: 10.1056/NEJMoa1507247.

44. Smolen JS, Kremer JM, Gaich CL, et al. Patient-reported outcomes from a randomised phase III study of baricitinib in patients with rheumatoid arthritis and an inadequate response to biological agents (RA-BEACON). Ann Rheum Dis 2017; 76 : 694-700, DOI: 10.1136/annrheumdis-2016-209821.

45. Genovese MC, Kremer JM, Kartman CE, et al. Response to baricitinib based on prior biologic use in patients with refractory rheumatoid arthritis. Rheumatology (Oxford) 2018; 57: 900908, DOI: 10.1093/rheumatology/kex489.
46. Dougados M, van der Heijde D, Chen YC, et al. Baricitinib in patients with inadequate response or intolerance to conventional synthetic DMARDs: results from the RA-BUILD study. Ann Rheum Dis 2017; 76: 88-95, DOI: 10.1136/annrheumdis-2016-210094.

47. Keystone EC, Taylor PC, Tanaka Y, et al. Patient-reported outcomes from a phase 3 study of baricitinib versus placebo or adalimumab in rheumatoid arthritis: secondary analyses from the RA-BEAM study. Ann Rheum Dis 2017; 76: 1853-1861, DOI: 10.1136/annrheumdis-2017-211259.

48. Fleischmann R, Schiff M, van der Heijde D, et al. Baricitinib, methotrexate, or combination in patients with rheumatoid arthritis and no or limited prior disease-modifying antirheumatic drug treatment. Arthritis Rheumatol 2017; 69: 506-517, DOI: 10.1002/art.39953.

49. van der Heijde D, Durez P, Schett G, et al. Structural damage progression in patients with early rheumatoid arthritis treated with methotrexate, baricitinib, or baricitinib plus methotrexate based on clinical response in the phase 3 RA-BEGIN study. Clin Rheumatol 2018; 37: 2381-2390, DOI: 10.1007/ s10067-018-4221-0.

50. Tanaka Y, Atsumi T, Amano K, et al. Efficacy and safety of baricitinib in Japanese patients with rheumatoid arthritis: Subgroup analyses of four multinational phase 3 randomized trials. Mod Rheumatol 2018; 28: 583-591, DOI: 10.1080/14397595.2017.1392057.

51. Posada MM, Cannady EA, Payne CD, et al. Prediction of transporter-mediated drug-drug interactions for baricitinib. Clin TransI Sci 2017; 10: 509-519, DOI: 10.1111/cts.12486. 